\title{
Berliner Gesellschaft für Psychiatrie und Nervenkrankheiten
}

\section{Sitzung yoлi 13. März 1899.}

Herr $\Lambda$ V. König: Ueber ,springende Pupillen” in einem Falle von cerebraler Kinderlähmung nebst einig'en Be-merkungen über die prognostische Bedeutung der ,sprin-genden Pupillen” bei normaler L· ichtreaction.

Der Vortragende fasst seine Ausführungen folgendermassen z $\alpha$-sarnmen:

1. Die ,springende Mydriasis” ist eine seltene Erscheinung. Siekommt bei normaler wie pathologischer Pupillenreaction vor. Beob-achtet wnrde sie bisher hauptsächlich bei organischen Erkrankungendes Central-Nervensystems, selten bei soldier functioneller Natur, undnur vereinzelt bei Nervenleidenden resp. sonst Gesunden.

Die prognostische Bedeutung der ,springenden” Mydriasis kommt nur bei normaler Pupillenreaction in Betracht, da bei jiatho-logischer Pupillenreaction dies*1 Thatsache allein die nöthige Directive geben wird.

Bei normaler Pupillenreaction und Fehlen von Symptomen eines organischen Nervenleidens ist das Auftreten der springenden M. nicht nothwendigerweise von abler Bedeutung.

Therapeutische Umschau.

409

4. Ein Vortäuschen der ,springenden” M. kann bei oberflächlicher Betrachtung vorhanden sein infolge wechselnden Einflusses zuund abnehmender Beleuchtung auf solche Pupillen, deren Weite und Reactionsintensität deutlich verschieden sind, besonders also bei einseitiger Pupillenstarre. $\pi$, ,

\& ' Pollack.

Therapeutische Umschau.

Die bisherigen Erfahrungen mit Holocain als lokales Anaestheticum bei Auge $\pi$ operationen. Die Versuche, ein wohlfeiles Ersatzmittel für Cocain zu finden, welches alle Vorteile dieses Anästhetikiims bietet ohne die Gefahr der bei Cocain gelegentlich auftretenden üblen Nebenwirkungen, sind nicht neu, aber alle bisher in Vorschlag gebrachten Mittel als: Eucain, Tropacocaín, Euphthalmin haben sicli schliesslich als mehr weniger ungeeignet erwiesen. Neuerdings scheint in dem Holocain ein wirklich wertvolles und in mancher Beziehung dem Cocain sogar überlegenes lokales Anästhetikum gefunden zu sein, das zur Anwendung in der Ophthal-mologie sich besonders emptiehlt.

3-4 Tropfen einer 1 prozentigen salzsauren Lösung von Holocain (chemisch ein Aethenylamidin) bewirken schon eine Minute nach erfolgter Einträufehmg in ein nicht entzündetes Auge eine totale Anästhesie der Cornea und eine so starke Herabsetzung der Bindehaut-sensibilität, $\bullet$ lass Berührung d. C. bulbi nicht mehr empfunden wird.

Die Hovuhaut bleibt dabei feucht und glänzend; Epithelvertrock-uung tritt nicht auf. Die Sparmung des Auges wird nicht beeinflusst, ebeuso $\Lambda$ venig die Pupille iiud die Akkoinodation.

Die Anästhesie bei einmaliger Instillation dauert 5 - 9 Minuten. 
Bei droimaliger Eintröpfelung von je 4 Tropfen in Intervallen von zirka 5 Minuten kann eine Anästhesie von durchschnittlich 30 Minuten erhalten werden. (Löwenstamm.)

Subjektiv mft Holocain nur ein scliwaches Gefühl von Brennen ähnlich wie Cocain hervor;

Rötung des Auges tritt dabei nicht auf.

Holocain wirkt in 1 prozentiger Lösung baktericid (Heinz und Schlösser), sodass eine

Sterilisierung der Lösungen nicht unbedingt erforderlích ist.

Am entzündeten Auge ist die Anästhesie vielleieht etwas geringer, jedenfalls aber so gross, dass galvanokaustische Eingriffe, Schlitzung der Cornea etc. schmerzlos ausgeívhrt werden können.

W. Wie Tierversuche ergeben haben, ist Holocain ein heftiges Krampfgift analog dem Strychnin. Infolgedessen verbietet sich vorerst seine Anwendung zu subkonjunktivalen lnjektionen. Bei einfacher Instillation sind bisher toxische Wirkungen niemals beobachtet worden. 